\title{
DISTRIBUTION OF THE MAGNETIC FORCE IN THE SURFACE LAYERS OF SUNSPOTS
}

\author{
J. JAKIMIEC \\ Astronomical Institute, Wroclaw University, Poland
}

\begin{abstract}
At the beginning the problem of constructing the three-dimensional magnetohydrostatic models of the photospheric layers in sunspots is discussed in some detail. It is pointed out that the construction of such models by solving the set of equations of magnetohydrostatics cannot be effectively carried out.

In order to solve the difficulties a suitable method of determining the distribution of the magnetic force in sunspots from measurements of the magnetic field has been worked out. Tentative results of the computations are presented.

General features of the distribution of the magnetic force in the photospheric layers of stable sunspots are discussed. It is pointed out that significant magnetic forces are necessary in the penumbra; they secure its transversal equilibrium, but are rather unimportant for its vertical structure. And it is quite probable that the magnetic field in the umbras of stable spots is nearly potential or force-free down to the photospheric level.
\end{abstract}

There are many observational data relevant to the photospheric layers of sunspots. Nevertheless, it is not easy to construct a complete model of these layers.

Such a complete model must be a three-dimensional one, because physical quantities in a sunspot vary in a horizontal as well as in the vertical direction. At present we are able to deal with the simplest such model only, viz., with the one which has the following properties:

(1) it is an average model, i.e. fluctuations connected with fine structures of sunspots are neglected;

(2) it is an axially symmetric model, i.e. the considerations are restricted to the sunspots of the most regular structure;

(3) it is a static model, i.e. systematic motions (Evershed effect) are ignored estimations show that the observed velocity field is of secondary importance for the over-all equilibrium of the considered layers of a sunspot (Jakimiec, 1965), and therefore a static model should provide a good approximation.

Figure 1 is intended to visualize the peculiar geometry of the considered region of a sunspot. Transverse dimensions of the region are much larger than its vertical thickness (in the figure the $z$-scale is even stretched). Moreover, the detailed shape of the region is not known from observations; only an estimation of the depression $\Delta z$ at the umbral-penumbral boundary can be obtained, from analysis of the Wilson effect. Thus the complete model of the photospheric layers of a sunspot must determine not only the distribution of the physical quantities in the region but also the geometry of the region. And the consistency between the geometry and the distribution of forces must be secured in the model.

It is rather obvious that the construction of such a model should be based on the ample experience gained already in constructing the average models of the undisturbed 
photosphere. It is known that the standard procedure in the case of the undisturbed photosphere falls into two main stages:

(1) Determination of the empirical temperature distribution $T(\tau)$, from the measurements of the limb darkening.

(2) Determination of the pressure distribution by integrating the equation of hydrostatic equilibrium:

$$
d p / d z=-\varrho g .
$$

Introducing the optical depth $\tau$ as a new independent variable,

$$
d p / d \tau=g / \kappa,
$$

and making use of the previously obtained $T(\tau)$ the integration can be effectively performed.

It will be easily understood that such a division into two stages should be retained also while constructing the model of the photosphere in a sunspot. From very precise

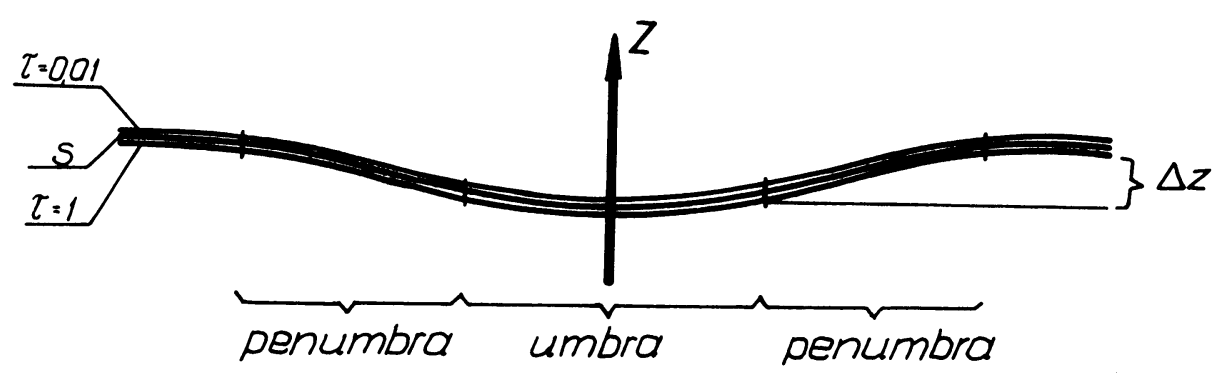

Fig. 1. Geometry of the photospheric layers in a sunspot, schematically. The probable shape of the surfaces $\tau=$ const is shown. $S$ is the surface, to which the measured magnetic field relates.

spectrophotometric observations the empirical temperature distributions $T(\tau ; r)$ for individual vertical columns of the photosphere in a spot could, in principle, be obtained. (Accuracy of the present observations is not as yet sufficient for that.)

The integration of the equations of magnetohydrostatic equilibrium to obtain the pressure distribution (together with the distribution of the magnetic field) would be now a natural generalization of stage 2) for our case. The equations form a set of non-linear partial differential equations; necessary boundary conditions should be supplied by observational data. Unfortunately, this direct way of determining the pressure distribution cannot be utilized effectively. The main reasons for that, without going into details, are as follows:

(a) in our three-dimensional case the change of variable $z \rightarrow \tau$ cannot be effectively carried out and therefore the temperature distribution $T(\tau ; r)$ cannot be directly used in the integration;

(b) the available observational data fail to provide appropriate boundary conditions; 
(c) the shape of the surface $S$, to which the boundary conditions (observations) relate, is not known a priori.

Moreover, the theory of the boundary value problems for such non-linear elliptical systems of equations is not yet worked out in detail.

Because of this state of affairs, the present author undertook the investigation of the problem of determining precise values of the magnetic force in sunspots from measurements of the magnetic field. It can be easily understood that the possibility of determining the distribution of the magnetic force, i.e. magnetic terms in the equations of magnetohydrostatic equilibrium, with an appropriate accuracy would open the way for determining the pressure distribution in the model of the photosphere in a sunspot.

As it is, a promising method of determining magnetic forces in sunspots has just been worked out (Jakimiec, 1970). It is mathematically expedient to consider the problem in terms of determining electric currents in a sunspot; then the main difficulty consists in determining the azimuthal component $j_{\varphi}$ of the current which is of the prevailing importance for our problem. The currents are determined by solving a boundary value problem for differential equations. The measured magnetic field is used as the boundary condition $(\mathbf{H})_{S}$.

The idea of detecting the currents situated in the region above $S$ can be summarized as follows: The presence of the currents in the region will have such an effect on the measured field $(\mathbf{H})_{s}$ that it will not fit to any potential field, and from the deviations of $(\mathbf{H})_{S}$ from the potential case inferences about the currents can be drawn. In order for the solution of the problem to be determined uniquely, use is made of the fact that in the considered region the gas pressure, and therefore also magnetic forces, decrease steeply with height. (For mathematical details see the paper cited above.)

The method has, inter alia, the following advantage: It allows to solve relatively easily the difficulty that the detailed shape of the surface $S$, to which the measurements of the magnetic field relate, is not known from observations. The shape is determined as an additional unknown function $z_{0}(r)$ by making use of the fact that it must be closely related to the distribution of the magnetic forces on the surface: The greater the slope of the surface $S$ at a given point, the greater, on the average, should be the radial pressure gradient $\partial p / \partial r$, and therefore also the radial magnetic force $F_{r}$, at the point. In the computations actually performed this relation was approximated by the proportionality:

$$
d z_{0}(r) / d r=K\left(F_{r}\right)_{S},
$$

where $z_{0}(r)$ describes the shape of the surface $S$.

The detailed calculations have been carried out making use of the observational data of Stepanov and Gopasjuk (1962). Figure 2 shows the obtained distribution of the radial component $F_{r}$ of the magnetic force across the sunspot. The obtained shape of the surface $S$ is shown in Figure 3. It is seen that it agrees with the traditional picture of the depression in a sunspot (gradual slope in the region of the penumbra and flat bottom in the umbra).

Because present-day measurements of the magnetic field in sunspots may be subject 
to large systematic errors (see, e.g., Severny, 1967), the results should be considered as rather tentative ones. It can be estimated that in order to obtain reliable determination of the magnetic forces of the relevant order of magnitude it is necessary to have measurements of the individual components of the magnetic field with an accuracy of $\pm 50 \mathrm{G}$. The requirements do not seem to be excessive ones and they probably will be met in the near future.

Thus, there is, indeed, a real possibility of determining reliably the regular magnetic forces in the surface layers of sunspots from the measurements of the magnetic field, provided that main systematic errors of the measurements will be eliminated.

However, basing on the experience gained in the above calculations, some conclu-

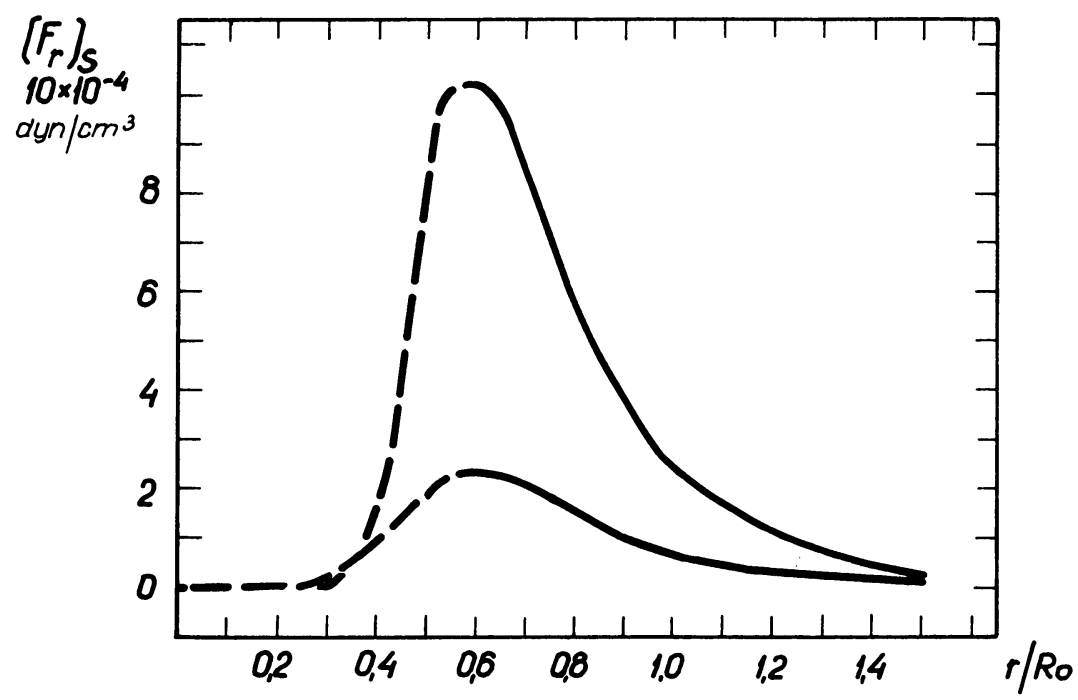

Fig. 2. The obtained distribution of the radial magnetic force $F_{r}$ on the surface $S$ in the investigated spot. Two variants of the computations corresponding to extreme values of the rate of decrease of the magnetic force with height are shown. The dashed lines are drawn for the region of the umbra where measurements of the magnetic field are the most uncertain. $R_{0}$ is the radius of the spot.

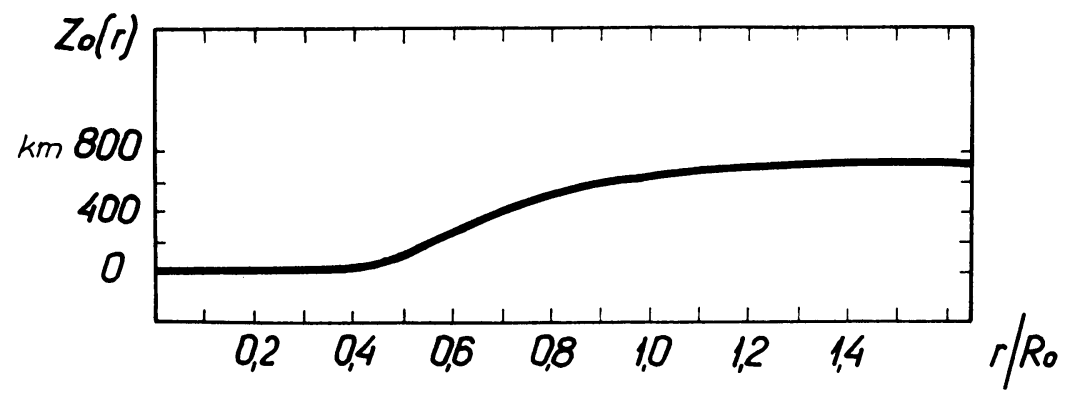

Fig. 3. The shape of the surface $S$ obtained in the computations. (The $z$-scale has been stretched.) 
sions about the distribution of the magnetic forces in the layers of sunspots can assuredly be drawn also at present.

First of all, if we start with the rather well-established fact that in a large spot the photosphere at the umbral-penumbral boundary is depressed some $700-1000 \mathrm{~km}$ below the undisturbed photosphere, we are forced to conclude that significant radial magnetic forces $F_{r}>0$ are necessary for the equilibrium of the penumbral photosphere. Moreover, from this fact reliable estimation of the mean value of $F_{r}$ in the penumbra can be made. The relevant equations are:

$$
F_{r}=\frac{\partial p}{\partial r} ; \quad \frac{\partial p}{\partial r}=-\frac{\partial p}{\partial z} \tan \zeta
$$

where $\zeta$ is the angle between the $z$-direction and the vector $(-\operatorname{grad} p)$ at a given point.

Characteristic values of $\partial p / \partial z$ at the level of line formation in the penumbra can be taken from the empirical model of the penumbral photosphere, calculated on the assumption of hydrostatic equilibrium (see remarks below): $|\partial p / \partial z| \sim 10^{-3} \mathrm{dyn} \mathrm{cm}^{-3}$. (The sub-hydrostatic model of Makita (1963) yields in fact the same order of magnitude of this quantity - cf. Jakimiec, 1970.)

As a characteristic value of $\zeta$ the mean slope of the solar surface in the penumbra can be taken:

Hence

$$
\tan \zeta \approx \frac{\text { depression } \Delta z \text { in the sunspot }}{\text { width of the penumbra }} \sim 10^{-1} .
$$

$$
\left(\bar{F}_{r}\right)_{S} \sim 10^{-4}{\mathrm{dyn} \mathrm{cm}^{-3}}^{-3}
$$

It is worthwhile to note that the values of the force $F_{r}$ derived from the above mentioned measurements of the magnetic field are of the correct order of magnitude (see Figure 2).

As to the forces $F_{r}$ in the umbra, we can say only that the usually accepted shape of

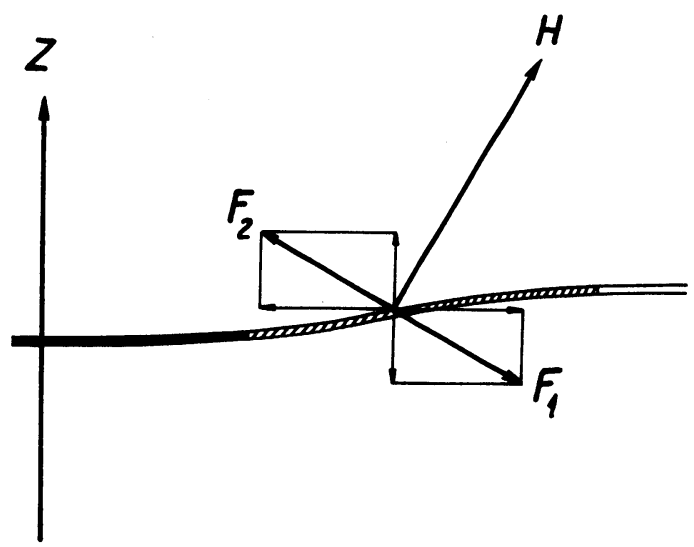

Fig. 4. Schematic diagram for discussing the direction of the vertical magnetic force $F_{z}$ in the penumbra. 
the depression in a sunspot, with flat bottom in the umbra, implies absence of significant regular radial forces $F_{r}$ there, i.e. the distribution of $F_{r}$ being of the type as shown on Figure 2.

Now several comments on the vertical component $F_{z}$ of the magnetic force in the penumbra are in order. On the schematic diagram (Figure 4) the typical inclination of the magnetic field $\mathbf{H}$ in the penumbra, as given by observations, is shown. The magnetic force $\mathbf{F}$ must be perpendicular to $\mathbf{H}$, so its projection on the plane of the diagram will be directed along $F_{1}$ or $F_{2}$. On the other hand, it has been shown in the previous considerations that the radial component $F_{r}$ of the magnetic force in the penumbra should be directed outwards $\left(F_{r}>0\right)$. Thus the case $F_{1}$ should take place and consequently the vertical component $F_{z}$ should be directed downwards $\left(F_{z}<0\right)$.

We see that it is rather difficult to expect regular magnetic forces supporting the solar atmosphere in the penumbra. The forces tend to increase effective gravitation, i.e. to compress the atmosphere. Nevertheless, the calculations show that $F_{z}$ is rather small in comparison with gravity, so that the deviations of the pressure from the distribution corresponding to the hydrostatic equilibrium do not exceed $10-20 \%$.

To summarize, the best overall self-consistent model of the photosphere in a sunspot, which can be proposed at present, would be the following one: Significant magnetic forces are present in the penumbra, they secure its transversal equilibrium, but are rather unimportant for its vertical structure. Hence also in the penumbra the vertical gas colums should be in nearly hydrostatic equilibrium. And it is quite probable that in the umbras of stable spots there are no significant regular magnetic forces, neither vertical, nor transversal ones. In other words, the smoothed magnetic field in there would be nearly potential or force-free down to the photospheric level.

\section{References}

Jakimiec, J.: 1965, Acta Astron. 15, 145.

Jakimiec, J.: 1970, Astron. Zh. 47, 520.

Makita, M.: 1963, Publ. Astron. Soc. Japan 15, 145.

Severny, A. B.: 1967, Izv. Krymsk. Astrofiz. Obs. 36, 22.

Stepanov, V. E. and Gopasjuk, S. I.: 1962, Izv. Krymsk. Astrofiz. Obs. 28, 194.

\section{Discussion}

Musman: What in your model is the difference in gas pressure between the center of a sunspot and the surrounding photospere at the same level.

Jakimiec: The depth of the sunspot is taken to be $700-1000 \mathrm{~km}$. Thus the total transverse pressure differences at the level of the umbral photosphere are of the order of $10^{6} \mathrm{dyn} \mathrm{cm}^{-2}$. The essential point here is that the pressure differences are equilibrated by magnetic forces in the region of the penumbra (mainly below the visible penumbra - cf. Figure 1).

Maltby: Could you comment on the stability of your sunspot model. Does not the energy equation enter in your discussion at all?

Jakimiec: Concerning the first question: the considerations concern only the thin layer of a sunspot (cf. Figure 1) and the problem of stability cannot be investigated for such a restricted region of the sunspot. But we need not trouble ourselves very much about the stability problem in the above considerations, because: (1) all the considerations concern the most stable, very slowly varying sunspots, 
(2) the stability of a sunspot as a whole is determined by the situation in the deeper layers and it is quite improbable that inaccuracies of a model of the considered photospheric layers could have any significant effect on the stability of the whole sunspot.

Concerning the second question: the equation of energy does not enter here because we use an empirical temperature distribution $T(\tau, r)$, which presents a ready (approximate) solution of the problem of the energy transport in the layers considered. This is quite analogous to the case of the empirical model of the undisturbed photosphere. 